\title{
Hemiageusia resulting from a cavernous haemangioma in the brain stem
}

A

A 34 year old man without any history of neurological diseases noted a sudden loss of taste on the right half of his tongue. At the same time he complained of a mild headache and an impaired coordination of the right hand, which resolved completely within three hours. On neurological examination the patient was unable to distinguish sweet, bitter, salty, and sour substances on the right half of his tongue. Movement of the tongue was normal and examination of pharyngeal and vocal cord structures showed no abnormality. There were no other neurologic deficits. Routine laboratory tests and analysis of CSF were normal. Visual evoked potentials, brainstem auditory evoked potentials, somatosensory evoked potentials after both median and posterior tibial nerve stimulation and transcranial magnetic stimulation to both hands were normal.

The MRI revealed a T2-hyperintense and $\mathrm{Tl}$-isointense right paramedian lesion on the floor of the fourth ventricle at the lower pontine level with high signal intensity on diffusion weighted sequences. MR angiography showed no abnormality. Additional contrast enhanced T1-SE and T2-FEE sequences revealed a cavernous haemangioma in association with a developmental venous anomaly (DVA) as the source of a pontine bleeding (fig l).

An operative intervention was rejected for the time being because of the high complications of surgery in this vulnerable area in comparison to the relative small chance of recurrent bleedings. The patient reported a spontaneous improvement of his taste 2 months later.

Impairment of taste, or a-/dysgeusia, includes a variety of possible causes. Besides the affection of the peripheral gustatory structures, which are mostly related to infections, drug side effects, and tumours, dysgeusia can also, but much less frequently, be due to a lesion in central taste pathways, including patients with multiple sclerosis, ${ }^{1}$ ischaemic infarction, ${ }^{2}$ and intracranial haemorrhage.

We report the rare case of isolated hemiageusia due to a spontaneous haemorrhage in the ipsilateral solitary tract resulting from a cavernous haemangioma. Our findings are in accordance with previous studies that report unilateral pontine lesions resulting in ipsilateral gustatory deficits. The total lack of sensory deficits in our patient suggests that the lemnicus medialis was not affected. Impaired coordination of the right hand might be explained by affection of the right inferior cerebellar peduncle (olivocerebellar tract).

Cerebral cavernomas were notoriously difficult to diagnose before the advent of MRI scanners. DVAs are frequently (up to $33 \%$ ) associated with a cavernoma, but are them-
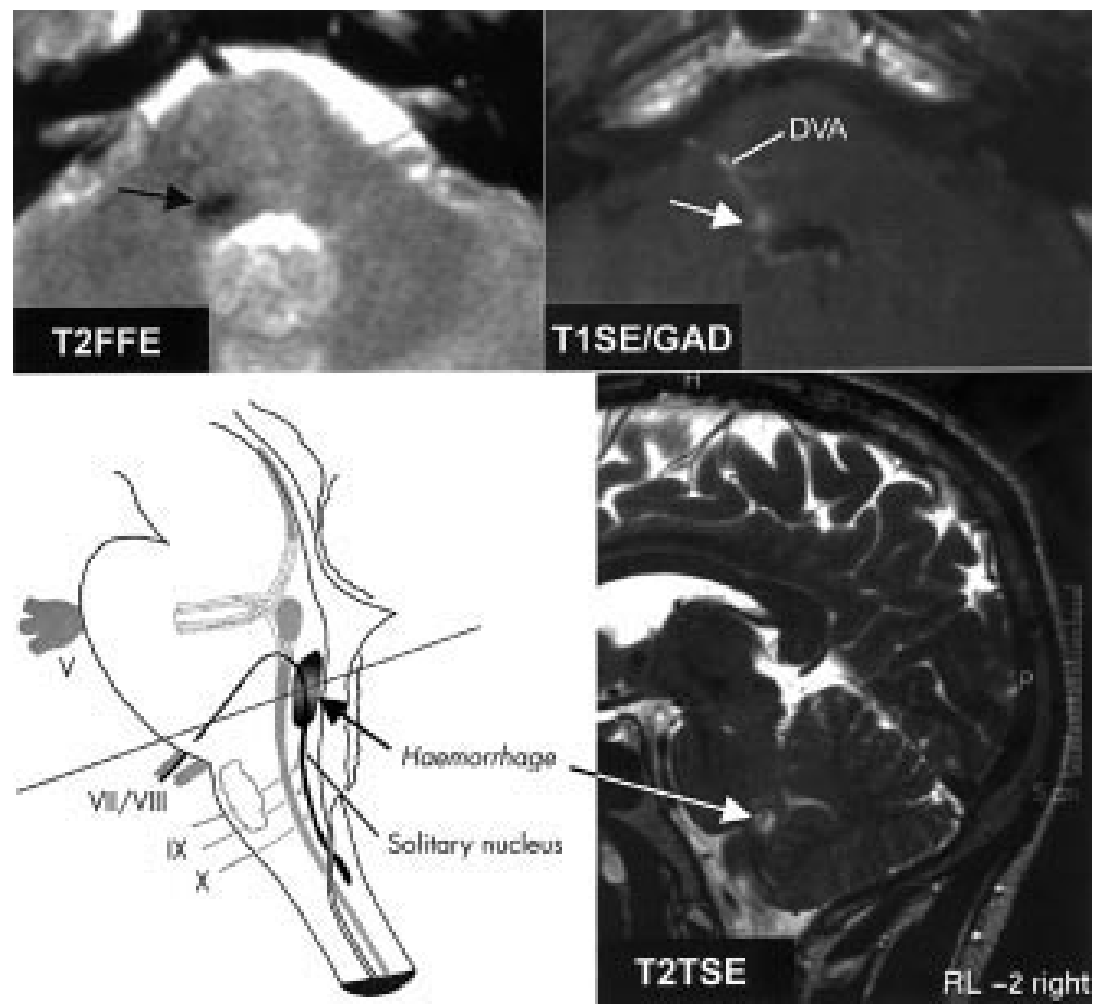

Figure 1 MRI findings (black and white arrows indicate haemorrhage). Scheme modified after Duus P, Neurologisch-topische Diagnostik. Stuttgart; New York: Thieme 2001.

selves an unlikely cause of cerebral haemorrhage. ${ }^{4}$ Thus the detection of a DVA should lead to a search for an associated cavernoma. The annual spontaneous bleeding rate of cavernomas is estimated to be in a range of $2-3 \% .^{5}$ T2-FFE sequences are more sensitive for the haemosiderin induced susceptibility artefacts of a cavernous haemangioma than standard SE/TSE sequences. Our case confirms also the importance of choosing appropriate MRI sequences in clarifying unusual brainstem lesions.

J Weidemann Department of Neuroradiology Universitätsklinikum, Technical University of Aachen, Pauwelsstr. 30, D-52074 Aachen, Germany

R Sparing

Department of Neurology, Technical University of Aachen, Germany

Correspondence to: $\mathrm{Dr} J$ Weidemann juergen-weidemann@t-online.de

\section{References}

1 Goto N, Yamamoto T, Kaneko M, et al

Primary pontine haemorrhage and gustatory disturbance: Clinicoanatomic study. Stroke 1983;14:507-11.

2 Lee BC, Hwang SH, Rison R, et al. Central pathway of taste: clinical and MRI study. Eur Neurol 1998;39:200-3.

3 Pascual-Leone A, Altafullah I, Dhuna A. Hemiageusia: an unusual presentation of multiple sclerosis. J Neurol Neurosurg Psychiatry 1991;54:657.

4 Kupersmith MJ, Kalish $\mathrm{H}$, Epstein F, et al. Natural history of brainstem cavernous malformations. Neurosurgery 2001;48:47-54.

5 Töpper $\mathbf{R}$, Jürgens $E$, Reul J, et al. Clinical significance of intracranial developmental venous anomalies. J Neurol Neurosurg Psychiatry 1999;67:234-8. 